CONGENITAL HEART DISEASE

\title{
Isolated left ventricular apical hypoplasia: a new congenital anomaly described with cardiac tomography
}

\author{
M Fernandez-Valls, M B Srichai, A E Stillman, R D White
}

See end of article for

authors' affiliations

.....................

Correspondence to: Dr R D White, Section of Cardiovascular Imaging, Desk Hb6, Cleveland Clinic Foundation, 9500 Euclid Avenue, Cleveland, Ohio 44195, USA; whiter@ ccisdl.ccf.org

Accepted 16 October 2003
Heart 2004;90:552-555. doi: 10.1136/hrt.2003.010637

Objective: To describe cardiac tomography findings of an apparently new, presumably congenital, left ventricular (LV) abnormality noted consistently in three patients.

Patients: Three patients presenting with non-specific symptoms including fatigue, shortness of breath, or chest discomfort were evaluated with cardiac tomography for cardiac structure and function.

Results: Findings from the three patients were very similar: a truncated and spherical LV with abnormal diastolic and systolic function, invagination of fatty material into the myocardium of the defective LV apex, origin of a complex papillary network in the anteroapical LV, and an elongated right ventricle wrapping around the deficient apex.

Conclusions: Isolated LV apical hypoplasia is a unique, presumably congenital, cardiac anomaly that is an important condition to recognise.
M agnetic resonance imaging (MRI) is well established for assessing congenital abnormalities of the heart in adults. ${ }^{1}$ Recent advances in cardiac computed tomography (CT), especially multidetector CT (MDCT), have increased its ability to define congenitally abnormal structures. $^{2}$

Cardiac tomography, by MRI or MDCT, shows characteristic gross morphological and tissue changes of acquired ${ }^{3-7}$ or congenital $^{7-9}$ diseases of the left ventricle (LV). We describe cardiac tomographic findings of an apparently new, presumably congenital, LV abnormality noted consistently in three patients. The findings suggested isolated LV apical hypoplasia.

\section{METHODS}

Patient population

Clinical description

The three patients were a 22 year old women of European descent (case 1), a 46 year old woman of Middle Eastern descent (case 2), and 26 year old man of European descent (case 3). Presenting symptoms were relatively mild and nonspecific and included fatigue, shortness of breath, or chest discomfort. Personal and family medical histories were noncontributory. Physical examination found systolic murmurs in all three patients.

Prior diagnostic evaluation

ECGs showed normal sinus rate and rhythm, right axis deviation $\left(110-130^{\circ}\right)$, and low precordial voltages with poor $\mathrm{R}$ wave progression. Transthoracic echocardiograms showed variable degrees of LV systolic dysfunction (case 1: mild; case 2: mild to moderate; case 3: moderate to severe), always with bulging of the interventricular septum towards the right ventricle (RV), and variable degrees of mitral abnormality (case 1: moderate regurgitation; case 2: prolapse; case 3: none). Left heart catheterisation showed increased LV end diastolic pressures in one patient (case 1: 30-35 mm Hg) but normal indices in another (case 2); in both patients, no coronary artery abnormality was seen on selective angiography.

For all three patients, the primary diagnosis after the aforementioned evaluations and before cardiac tomography was idiopathic cardiomyopathy. All assessments of familial, metabolic, infiltrative, infectious, and inflammatory cardiomyopathy were negative.

\section{Cardiac tomography techniques \\ MRI techniques}

MRI was done with a phased array coil and standard ECG based referencing (Symphony or Sonata, Siemens Medical Systems, Erlangen, Germany).${ }^{10}$ After LV axes were identified with scout imaging, diagnostic imaging was performed. Anatomical evaluation was done with segmented $\mathrm{k}$ space "dark blood" spin-echo sequences with or without fat saturation. ${ }^{10}$ Dynamic evaluation was done with "bright blood" (cine) segmented k space gradient echo imaging incorporating view sharing to improve temporal resolution. ${ }^{10}$ Dynamic tissue tagged ( $8 \mathrm{~mm}$ grid stripe separation) myocardial imaging with a corresponding sequence (fast low angle shot based spatial modulation of magnetisation $)^{10}$ was used with two patients (cases 2 and 3 ).

\section{MDCT techniques}

For cases 1 and 3, CT was done with four detector (Volume Zoom; gantry rotation $500 \mathrm{~ms}$, temporal resolution $250 \mathrm{~ms}$ ) or 16 detector (Sensation 16; gantry rotation $420 \mathrm{~ms}$, temporal resolution $210 \mathrm{~ms}$ ) MDCT systems (Siemens Medical Systems, Forchheim, Germany). ${ }^{7}$ Retrospectively ECG gated spiral imaging (collimation $4 \times 1 \mathrm{~mm}$, increment $0.5 \mathrm{~mm}$, thickness $1.25 \mathrm{~mm}$ or collimation $12 \times 0.75 \mathrm{~mm}$, increment $0.5 \mathrm{~mm}$, thickness $1.00 \mathrm{~mm}$ ) was used. Scanning followed a proper delay determined after power injection of an intravenous test bolus $(20 \mathrm{ml}$ at $2.5 \mathrm{ml} / \mathrm{s})$ of non-ionic contrast medium (Ultravist 300, Berlex, Pine Brook, New Jersey, USA) with complete intracardiac enhancement during diagnostic imaging with a larger contrast volume (100-150 $\mathrm{ml}$ at $2.5 \mathrm{ml} / \mathrm{s}$ ).

The MDCT data were reviewed on a workstation (Wizard, Siemens Medical Systems, Erlangen, Germany) with interactive scrolling, multiplanar reconstructions, and maximum intensity projections.

Abbreviations: CT, computed tomography; LV, left ventricular; MDCT, multidetector computed tomography; MRI, magnetic resonance imaging; $\mathrm{RV}$, right ventricle 


\section{RESULTS}

\section{Cardiac tomography findings} MRI findings

MRI showed the same morphological abnormalities in all three patients: a truncated appearance of the spherical (rather than normal conical) LV with bulging of the interventricular septum towards the RV; invagination of fatty material contiguous with epicardial fat into the area normally occupied by the LV apex; origin of a complex papillary muscle network in the flattened anteroapical (rather than anterolateral and posteromedial) region; and elongation of a normally functioning RV wrapped around the deficient LV apex (fig 1).

Visual assessment of the dynamic MRI data showed that the patients had abruptly limited late diastolic filling in a restrictive pattern and decreased contractility (fig 2). Additionally, visualisation of significant mitral regurgitation was noted in only one patient (case 1: moderate).

\section{MDCT findings}

A defective LV apex was visualised on MDCT. Better seen on MDCT than on MRI was the extent to which fatty material replaced the deficient apical myocardium, especially towards the distal interventricular septum (fig 1).

\section{DISCUSSION}

MRI and MDCT showed findings of this apparently new anomaly, isolated LV apical hypoplasia: (1) a truncated and spherical LV configuration with rightward bulging of the interventricular septum, associated with impaired late diastolic filling and systolic mechanics; (2) replacement of the LV apical myocardium, especially along the distal interventricular septum, with fatty material contiguous with epicardial fat; (3) origin of a complex papillary muscle network in the flattened anterior apex (but with significant regurgitation in only one of three patients); and (4) elongation of the RV wrapping around the deficient LV apex. These findings account for the clinical presentation and initial diagnostic evaluation suggesting cardiomyopathy.

\section{Differential diagnoses: acquired lesions}

Acquired processes were considered as potential causes of this cardiac condition. However, as our patients were dissimilar in age, sex, and ethnic group, causes other than congenital seemed unlikely. Additionally, the morphological changes differed from those of myocarditis or ischaemic heart disease. While Chagas's disease often leads to LV involvement and may preferentially affect the apex, regions of fibrotic wall thinning commonly cause generalised dilatation with apical aneurysms. ${ }^{11}$ None of our patients had such changes or evidence of coronary abnormalities.

\section{Differential diagnoses: congenital lesions}

To our knowledge, no prior report matches this description of isolated LV apical hypoplasia. Acknowledging similarities to known conditions, we believe that this cardiac condition is unique.

It is clear that this condition is not hypoplastic left heart syndrome, which is characterised by underdevelopment of the aorta, the aortic valve, and the entire LV resulting from interrupted growth due to genetic influences or secondary to diminished flow to the developing LV. ${ }^{12}$ While hypoplastic left heart syndrome is associated with an atretic or stenotic
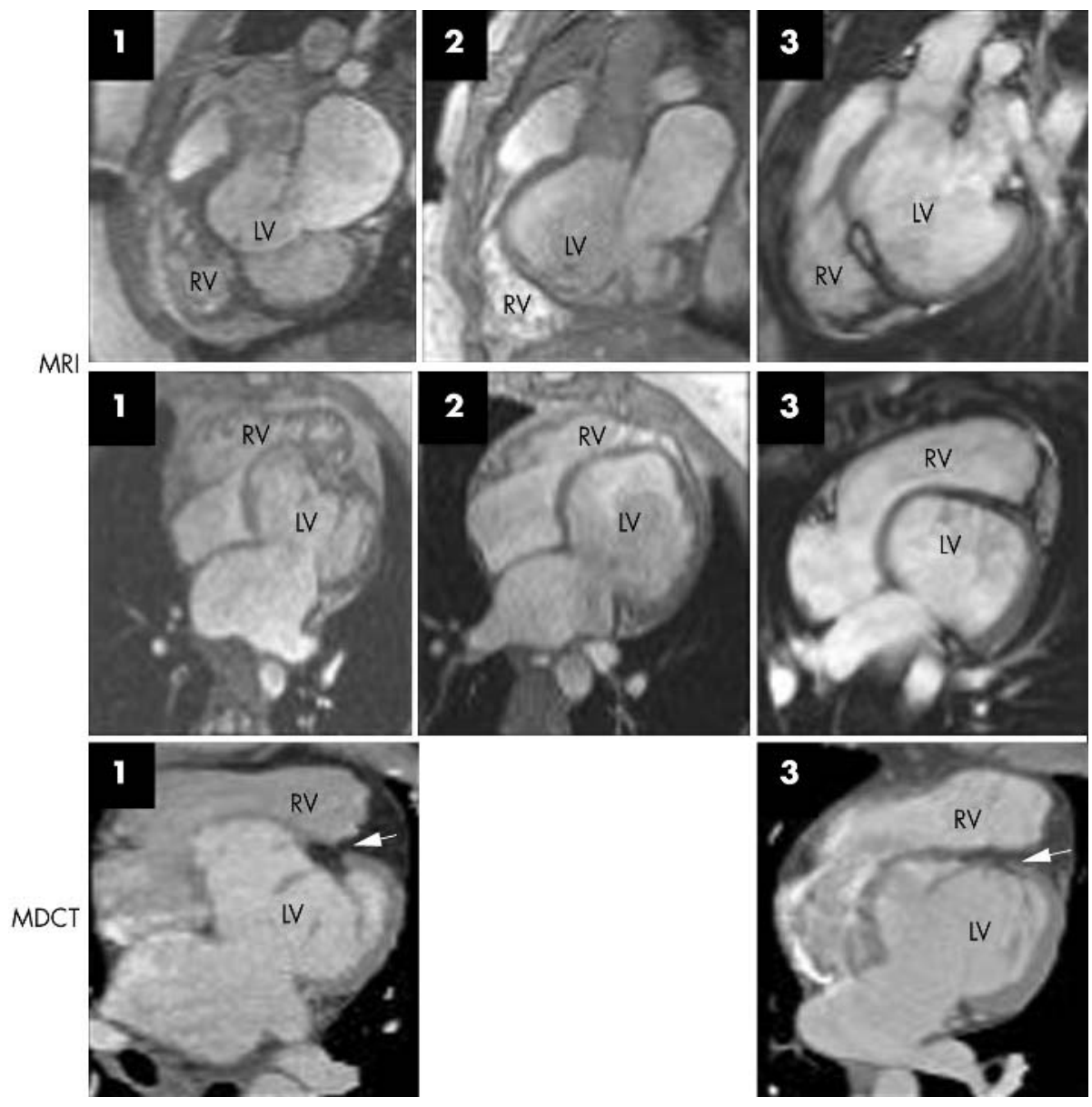

Figure 1 Cardiac tomography of isolated left ventricular (LV) apical hypoplasia. Corresponding LV oufflow tract and four chamber views from cine bright blood magnetic resonance imaging (MRI) in cases 1-3 (first and second rows) and corresponding four chamber views from multidetector computed tomography (MDCT) in cases 1 and 3 (third row) all show a spherical, truncated LV with bulging of the interventricular septum towards the right ventricle (RV), invagination of fatty material (arrows) contiguous with epicardial fat into the defective LV apex, origin of a complex papillary muscle network in the flattened anterior apex, and elongation of the RV, which wraps around the deficient $\mathrm{LV}$ apical region. 

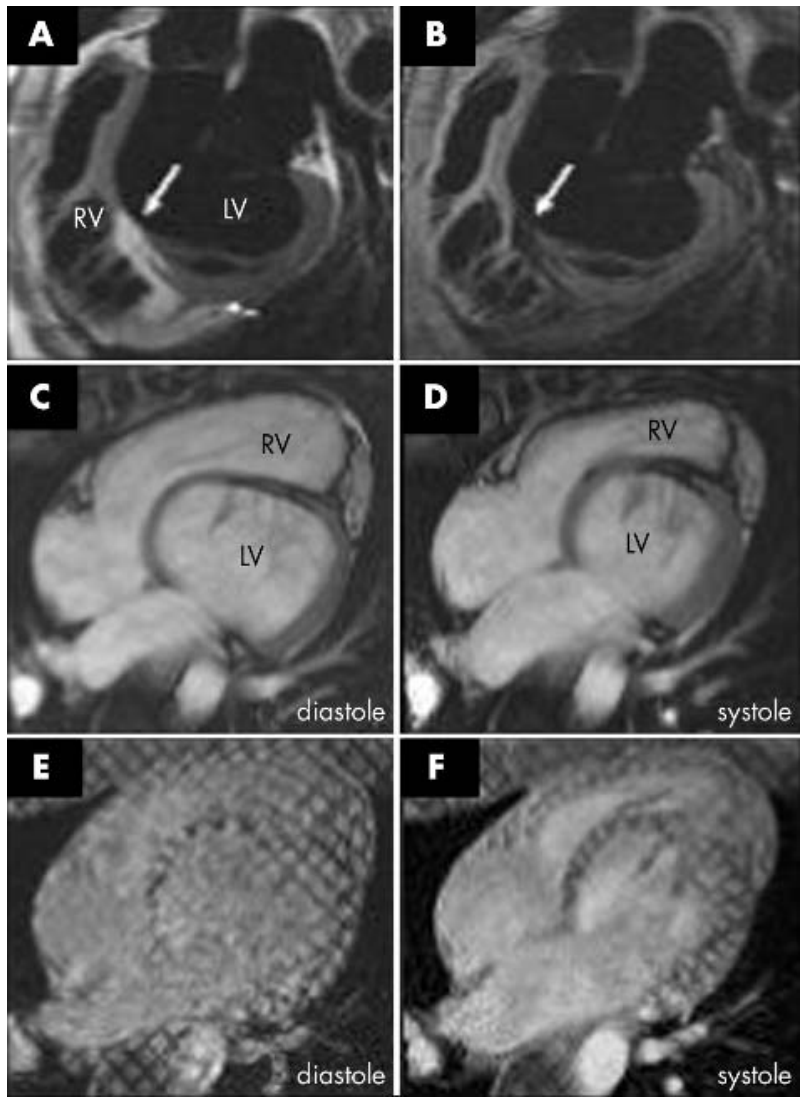

Figure 2 Comprehensive evaluation of isolated LV apical hypoplasia by cardiac MRI. Anatomical evaluation with dark blood imaging (A) without and then (B) with fat saturation confirms the fatty nature of the material (large arrows) contiguous with epicardial fat (small arrow) and extending into the truncated LV apex. Dynamic evaluation with $(C, D)$ dynamic bright blood and $(E, F)$ tissue tagged imaging shows the spherical shape of the restrictive LV in late diastole and lack of systolic myocardial deformation due to impaired contractility, especially in the interventricular septum.

mitral valve $95 \%$ of the time, ${ }^{12}$ our patients had mature valves and malformation was limited to the LV apex.

Non-compaction of the LV results from arrested endomyocardial morphogenesis leading to dysfunction. ${ }^{13}$ It is characterised by a diffusely dilated LV with a heavily trabeculated endocardium, unlike the configuration found in our patients.

Congenital LV aneurysm, an idiopathic anomaly of the endocardium and myocardium, ${ }^{14}$ and LV diverticulum, part of an idiopathic syndrome with multiple defects, ${ }^{15}$ also appear to be different entities. As well as resulting in different LV morphologies featuring an elongated (as opposed to truncated) LV and involvement of the papillary muscles and surrounding myocardium (as opposed to isolated involvement of apex), these conditions usually lead to severe, usually fatal, clinical presentations early in life due to associated intracardiac and extracardiac defects (as opposed to relatively healthy adult presentations). ${ }^{15}$

Congenital LV dysplasia caused by abnormal endomyocardial morphogenesis in association with RV dysplasia is a generalised myocardial disease with predominantly RV involvement, ${ }^{16}$ which none of our patients had. In previously described cases of isolated LV dysplasia, in one case thinning of the posterior LV myocardial wall was notable with transmural fibrofatty replacement ${ }^{17}$ and in the other three there was extensive circumferential fibrofatty infiltration of predominantly LV myocardium causing cardiomegaly, systolic heart failure, and tachycardia. ${ }^{16} 18$ While fibrofatty myocardial replacement was seen in our patients, it was limited to the apex of the non-dilated dysmorphic LV, suggesting deficient apical development. In addition, in our patients dysfunction was predominantly diastolic, rather than systolic, and none of them had manifested any clinical signs or symptoms of tachyarrhythmias.

Isolated RV hypoplasia, a rare idiopathic condition characterised by RV underdevelopment with absence of its trabeculated apex, ${ }^{19}$ has RV features similar to those affecting the LV in our cases. Patients with this condition also have increased RV and RA pressures due to reduced compliance, not dissimilar to the diastolic dysfunction noted in our patients. ${ }^{19}$ However, their myocardial abnormalities are limited to the RV (as opposed to LV) and do not appear to share the fibrofatty morphology characterising our cases.

\section{Cardiac development and potential mechanisms}

Heart development begins around the third week of fetal life with formation of a cardiac tube that develops areas of constriction and dilatation to form primitive chambers. The primitive ventricle is partitioned in the fifth week with formation of the muscular interventricular septum. ${ }^{20}$ Defective LV apical development in our patients may be attributable to relatively inadequate $\mathrm{LV}$ to $\mathrm{RV}$ dilatation during partitioning, leading to the spherical LV with an elongated RV wrapping around its truncated apex as the interventricular septum continues to develop.

\section{Clinical management}

The natural history of this condition is uncertain, as our patients are relatively young and functional. However, standard treatment of their heart failure has been helpful. While none of them has evidence of tachyarrhythmias, concern for malignant arrhythmias in isolated LV apical hypoplasia seems appropriate.

\section{Conclusion}

Isolated LV apical hypoplasia is a unique, presumably congenital, cardiac anomaly that is an important condition to recognise.

\section{Authors' affiliations}

M Fernandez-Valls, M B Srichai, A E Stillman, R D Whitet, Department of Radiology (Section of Cardiovascular Imaging), Cleveland Clinic Foundation, Cleveland, Ohio 44195, USA

M B Srichai*, Department of Cardiovascular Medicine, Cleveland Clinic Foundation

†Also at Department of Cardiovascular Medicine, Cleveland Clinic Foundation

*Unrestricted training support from Berlex Laboratories, Pine Brook, New Jersey, USA.

\section{REFERENCES}

1 Hirsch R, Kilner PJ, Connelly MS, et al. Diagnosis in adolescents and adults with congenital heart disease: prospective assessment of individual and combined roles of magnetic resonance imaging and transesophageal echocardiography. Circulation 1994;90:2937-51.

2 Westra SJ. Spiral and ultrafast computed tomography for noninvasive cardiac imaging in children. West J Med 1996;165:55-6.

3 Sardanelli F, Molinari G, Petillo A, et al. MRI in hypertrophic cardiomyopathy: a morphofunctional study. J Comput Assist Tomogr 1993;17:862-72.

4 Masui T, Finck S, Higgins CB. Constrictive pericarditis and restrictive cardiomyopathy: evaluation with MR imaging. Radiology 1992;182:369-73.

5 Doherty NE 3rd, Fujita N, Caputo GR, et al. easurement of right ventricular mass in normal and dilated cardiomyopathic ventricles using cine magnetic resonance imaging. Am J Cardiol 1992;69:1223-8.

$6 \mathrm{Kim}$ RJ, Fieno DS, Parrish TB, et al. Relationship of MRI delayed contrast enhancement to irreversible injury, infarct age, and contractile function. Circulation 1999;100:1992-2002.

7 Ohnesorge B, Becker C, Flohr T, et al. Multi-slice CT in cardiac imaging: technical principles, clinical application and future developments. Munich: Springer, 2002. 
8 Kawano T, Ishii M, Takagi J, et al. Three-dimensional helical computed tomographic angiography in neonates and infants with complex congenital heart disease. Am Heart J 2000;139:654-60.

9 Choe YH, Kim YM, Han BK, et al. MR imaging in the morphologic diagnosis of congenital heart disease. Radiographics 1997;17:403-22.

10 Schvartzman PS, White RD. Magnetic resonance imaging. In: Topol EJ, ed. Textbook of cardiovascular medicine, 2nd ed. Philadelphia: Lippincott-Raven 2002.

11 Anselmi A, Pifano F, Suarez JA, et al. Myocardiopathy in Chagas' disease. I. Comparative study of pathologic findings in chronic human and experimental Chagas' myocarditis. Am Heart J 1966;72:469-81.

12 Freedom RM, Black MD, Benson LN. Hypoplastic left heart syndrome. In: Allen HD, Gutgesell HP, Clark EB, et al, eds. Moss and Adams' heart disease in infants, children, and adolescents, including the fetus and young adult. Philadelphia: Lippincott Williams \& Wilkins, 2001:1011-26.

13 Agmon Y, Connolly HM, Olson L, et al. Noncompaction of the ventricular myocardium. J Am Soc Echocardiogr 1999;12:859-63.
14 Papagiannis J, Van Praagh R, Schwint O, et al. Congenital left ventricular aneurysm: clinical, imaging, pathologic, and surgical findings in seven new cases. Am Heart J 2001;141:491-9.

15 Edgett JW Jr, Nelson WP, Hall RJ, et al. Diverticulum of the heart: part of the syndrome of congenital cardiac and midline thoracic and abdominal defects. Am J Cardiol 1969;24:580-3.

16 DePasquale C, Heddle W. Left sided arrhythmogenic ventricular dysplasia in siblings. Heart 2001;86:128-30.

17 Okabe M. Fukuda K, Nakashima Y, et al. An isolated left ventricular lesion associated with left ventricular tachycardia: arrhythmogenic "left" ventricular dysplasia? Jpn Circ J 1995;59:49-54.

18 Collett B, Davis G, Rohr W. Extensive fibrofatty infiltration of the left ventricle in two cases of sudden cardiac death. J Forensic Sci 1994;39:1 182-7.

19 VanDerHauwaert LG, Michaelsson M. Isolated right ventricular hypoplasia. Circulation 1971:44:466-74.

20 Moore KL. The cardiovascular system. In: Moore KL, ed. The developing human: clinically oriented embryology. Philadelphia: WB Saunders, 1988

\section{IMAGES IN CARDIOLOGY}

\section{Left ventricular pseudoaneurysm following myocardial infarction}

A n asymptomatic 61 year old man with a history of coronary artery disease, including coronary artery bypass surgery, presented for routine examination after a lateral myocardial infarction treated by thrombolysis five months previously. Physical examination was unremarkable. Echocardiography revealed a dyskinetic cavity connected to the posterolateral wall which communicated freely with the left ventricle. Magnetic resonance imaging (below left) confirmed the diagnosis of a false aneurysm (A) of the left ventricle (LV) by demonstrating a large perforation of the free posterolateral wall (arrows). Invasive coronary angiography revealed a patent left internal mammary artery graft to the left anterior descending coronary artery. A venous bypass to the left circumflex coronary artery demonstrated a severe stenosis at the distal anastomosis. Open heart surgery was performed. The aneurysmal sac was excised and the defect (below right, arrows) was closed with a pericardial patch. The postsurgical period was uneventful.

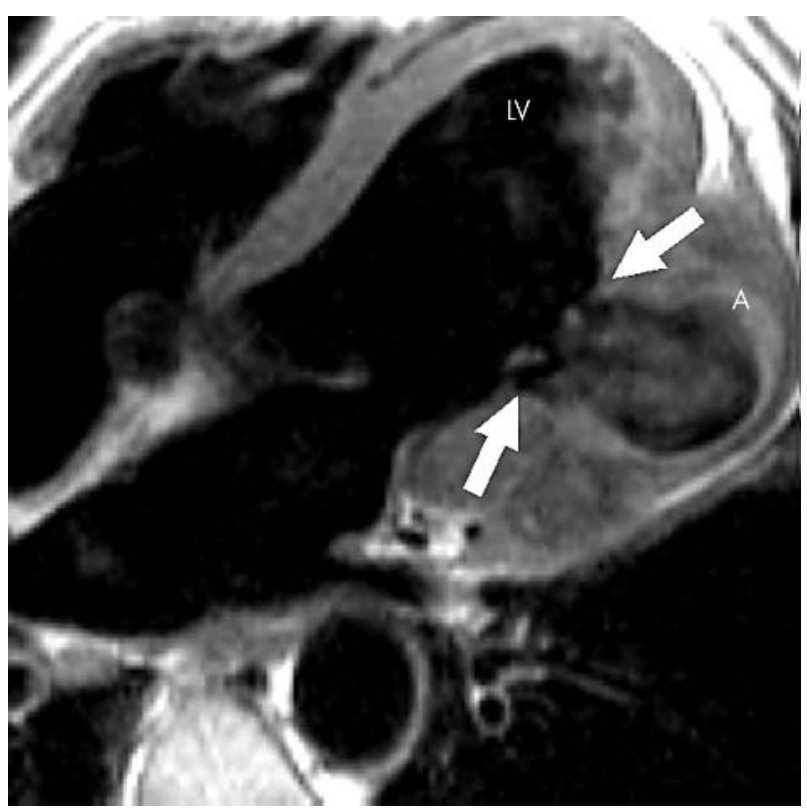

Rupture of the left ventricular free wall is a catastrophic complication of myocardial infarction, occurring in approximately $4 \%$ of patients with infarcts and about $23 \%$ of those suffering fatal infarcts. It is four to five times more common than septal rupture. Rarely, free wall rupture is contained by overlying, adherent pericardium, producing what has been termed a pseudoaneurysm or false aneurysm of the left ventricle. These pseudoaneurysms are often detected incidentally by echocardiography or other imaging modalities. Because of their propensity to rupture, emergency surgical intervention is recommended. The patient survived because the pericardium adhered to the epicardium as a consequence of bypass surgery six years earlier.

D Ropers, S Achenbach, S Pfeiffer dieter.ropers@rzmail.uni-erlangen.de

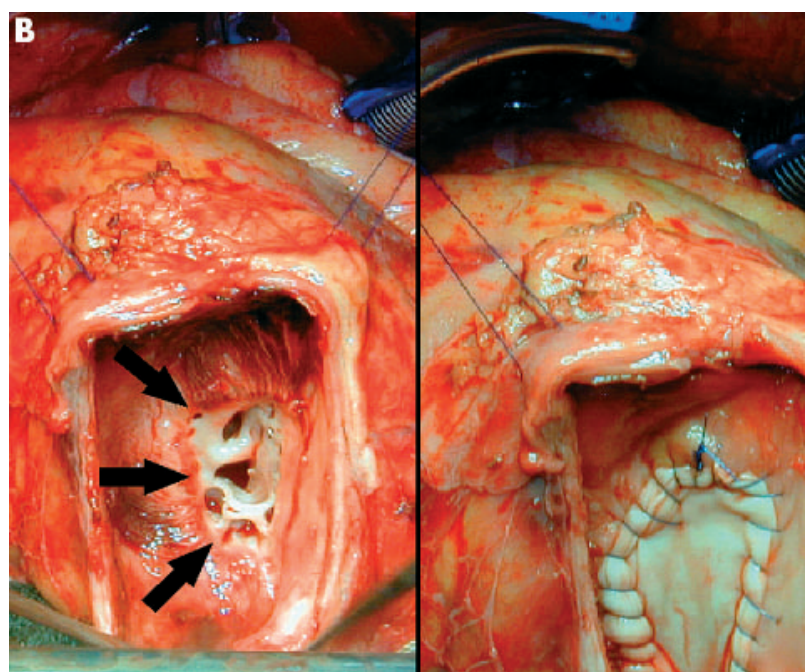

